\title{
Improvement of a Pound-Drever-Hall Technique to Measure Precisely the Free Spectral Range of a Fabry-Perot Etalon
}

\author{
Dong-Sun Seo $^{1}{ }^{*}$, Chongdae Park ${ }^{1}$, Daniel E. Leaird ${ }^{2}$, and Andrew M. Weiner ${ }^{2}$ \\ ${ }^{I}$ Department of Electronics, Myongji University, Yongin 449-728, Korea \\ ${ }^{2}$ School of Electrical \& Computer Engineering, Purdue University, W. Lafayette, IN 47907, USA
}

(Received February 24, 2015 : revised April 17, 2015 : accepted May 6, 2015)

\begin{abstract}
We examine the principle of a modified Pound-Drever-Hall (PDH) technique to measure the free spectral range of a Fabry-Perot etalon (FPE). The FPE's periodic transmission of phase-modulated light allows us to adopt a sampling theorem to develop a new relationship for the PDH error signal. This leads us to find the key parameters governing the measurement accuracy: the phase modulation index $\beta$ and the FPE finesse. Without any additional complexity for background noise reduction, we achieve a measurement accuracy of $0.5 \mathrm{ppm}$. The improvement is mainly attributed to the wide-band phase modulation approaching $\beta=10$, and partly to the use of both reflected and transmitted light from the FPE and good FPE finesse.

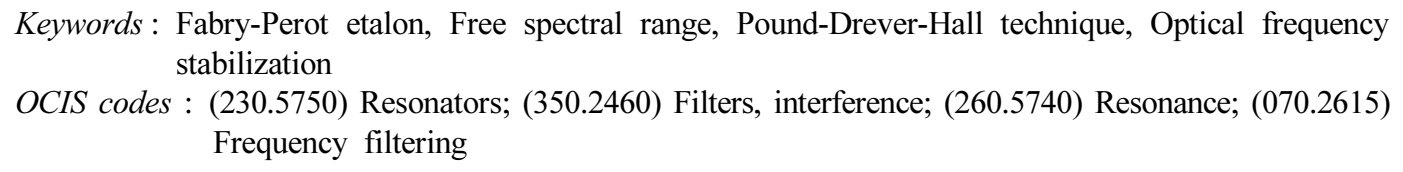

\section{INTRODUCTION}

Fabry-Perot etalons (FPEs) have many applications in the fields of optical communications and photonics. Highfinesse FPEs have been used for linewidth reduction or frequency stabilization of a semiconductor laser, optoelectronic oscillators, WDM filtering, and so on [1-3]. They have also been used for repetition-rate multiplication of pulsed lasers by spectral filtering [4-6]. Recently, an FPE was used inside a mode-locked laser cavity to stabilize or to lock the laser comb to the FPE transmission (TX) peaks [7, 8]. Having several hundred ultrastable comb lines locked to the FPE-TX peaks opened new application fields in WDM optical networks and photonics [7]. For diverse applications, precise measurement of the free spectral range (FSR) of an FPE is required. For example, to lock mode-locked spectral comb lines to intracavity FPE-TX peaks, the mode-locked laser frequency should stay within $1 \mathrm{ppm}$ (parts per million) of the FSR of the FPE [7, 8]. Conventionally, the FSR of an FPE has been measured by examining its transmission spectrum as the injected laser frequency is scanned [9]. This method is simple but has poor measurement accuracy, due to the resolution limit of the optical spectrum analyzer used to perform the measurement. Recently a new technique, a modified PoundDrever-Hall (PDH) method, was suggested [10], in which probe light was phase-modulated around the FSR of an FPE and injected into the FPE, and the reflected (or transmitted) light was monitored. The instantaneous amplitude of the reflected light gave information about the difference between the phase modulation $(\mathrm{PM})$ frequency $\left(\mathrm{f}_{\mathrm{pm}}\right)$ and FSR $\left(\mathrm{f}_{\mathrm{FSR}}\right)$. In Ref. [10] the authors achieved a measurement accuracy of $100 \mathrm{ppm}$. Ozdur et al., reported that the measurement accuracy was limited by unwanted static modulation of the background multiple-reflection noise [11]. To reduce the background noise, a slow time-varying (time-delay) modulation technique using a piezoelectric translator (PZT) was adopted, and resulted in a measurement accuracy improvement of two orders of magnitude to $1 \mathrm{ppm}$ [11]. Here, we demonstrate a simple setup that is similar to the scheme shown in [10], without the use of a PZT-based slow time-varying modulator loop to remove static modulation noise. In section II we examine the principle of the measurement technique and explain the error-signal generation with a simple conceptual diagram. We adopt a well-known sampling theorem and extract the key parameters governing the measurement sensitivity: the phase-modulation depth of the injected light

\footnotetext{
*Corresponding author: sdsphoto@mju.ac.kr

Color versions of one or more of the figures in this paper are available online.
} 
and the FPE finesse. In section III we discuss experimental results utilizing both the transmitted and reflected light of the FPE. We achieve a measurement accuracy of $0.5 \mathrm{ppm}$, a factor of two better than the previously reported accuracy, without requiring the additional complexity of a static modulation control loop. Finally, we present our conclusion in section IV.

\section{PRINCIPLE OF THE FSR MEASUREMENT}

The experimental setup to measure the FSR of an FPE is shown in Fig. 1 [12]. Probe light from a commercially available external cavity laser is scanned over an FPE-TX peak. The scanning frequency is set to $20 \mathrm{~Hz}$. Unlike in the conventional method, the probe light is strongly phase modulated (modulation index $\beta \sim 10$ ) at a frequency near the FSR of an FPE, and the phase-modulated light is injected to the FPE. The suggested measurement scheme relies on the alignments of the PM sidebands with respect to FPE transmission peaks, resulting in the spectral amplitude modulation of the sidebands. Details will be discussed later. In the specific measurement performed here, the FPE consists of 15-mm air-spaced concave (radius $50 \mathrm{~cm}$ ) and flat mirrors with 270 finesse and $0.01 \mathrm{ppm} / \mathrm{K}$ temperature dependence, respectively. The FSR provided by the vendor is $9.99 \mathrm{GHz}$. The reflected and transmitted light from the FPE are detected by separated photodetectors. The individual detector output for transmitted or reflected light is selected alternately by a switch, amplified by a radio-frequency (RF) amplifier, and mixed with the phase-modulator drive signal. To maximize the mixer output, we adjust the phase of the detector output by controlling an RF delay line. The mixer output, synchronized by the laser scanning frequency $(20 \mathrm{~Hz})$, is averaged and measured by a sampling scope. Hereafter we refer to the sampling scope trace as the "error signal", representing the frequency difference between the PM frequency $f_{p m}$ and $f_{F S R}$. The error signals for both transmitted and reflected light are obtained from the same circuit through the selectable switch. We monitor the error signal as we tune $f_{\text {pm }}$ around $f_{F S R}$.

Next we discuss the principle of error-signal generation. Strong sinusoidal $\mathrm{PM}$ at $\mathrm{f}_{\mathrm{pm}}$ generates a large number $(\sim 2$

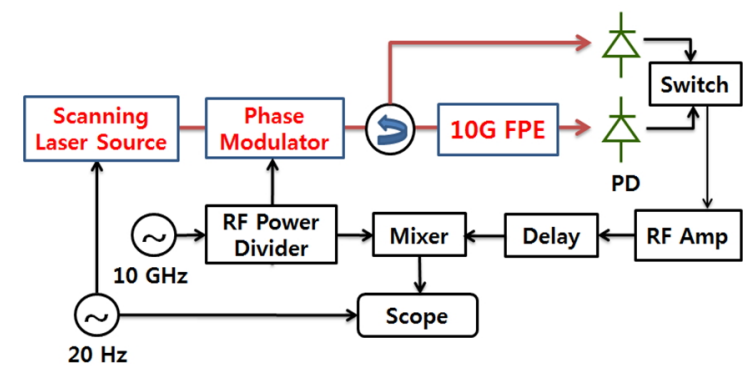

FIG. 1. Schematic diagram of the setup to precisely measure the FSR of an FPE (abbreviations can be found in the text). $\beta$ ) of upper and lower sidebands spaced by $f_{p m}$ around the unmodulated carrier frequency (here, the frequency of the probe light). The amplitude of the sidebands is a function of $\mathrm{J}_{\mathrm{n}}(\beta)$, the Bessel function of the first kind of order $n$ (positive for upper sidebands and negative for lower sidebands). Since the PM waveform occupies a large bandwidth $\left(\sim 2 \beta \times f_{\text {pm }}\right)$, this is called "wide-band" PM. Different alignments of the individual PM sidebands with respect to the FPE-TX peaks yield different losses, resulting in spectral amplitude modulation of the injected PM light. The amount of amplitude modulation, indicating the frequency difference $\delta=\mathrm{f}_{\mathrm{pm}}-\mathrm{f}_{\mathrm{FSR}}$, is detected by mixing the detector output and phase-modulator drive signal. To be explicit about the measurement principle, we will consider following three cases $(\delta>0, \delta=0$, and $\delta<$ 0 ) assuming that the PM light has four sidebands (order $n$ $= \pm 1$ and \pm 2 ).

\section{1. $\delta>0\left(\mathbf{f}_{\mathrm{pm}}>\mathbf{f}_{\mathrm{FSR}}\right)$}

In this case, the spacing between the PM sidebands is greater than $\mathrm{f}_{\mathrm{FSR}}$. Then the PM sidebands may be determined with respect to the FPE transmission peaks, as shown by the arrows in Fig. 2(a). By scanning the optical (carrier) frequency $v$ of the probe light, we can shift the spectrum groups from the pink to black to blue arrows. At a relatively low carrier frequency ( $v_{\mathrm{L}}$, pink arrows), the upper sidebands are enhanced but the lower sidebands are suppressed, after passing the FPE. This frequency-dependent loss induces the envelope (or amplitude) modulation at $\mathrm{f}_{\mathrm{pm}}$ for the injected PM light. For clarity of the envelope modulation, we adopt a well-known sampling theorem. All of the PM sidebands shown in Fig. 2 are then mapped on a single FPE-TX

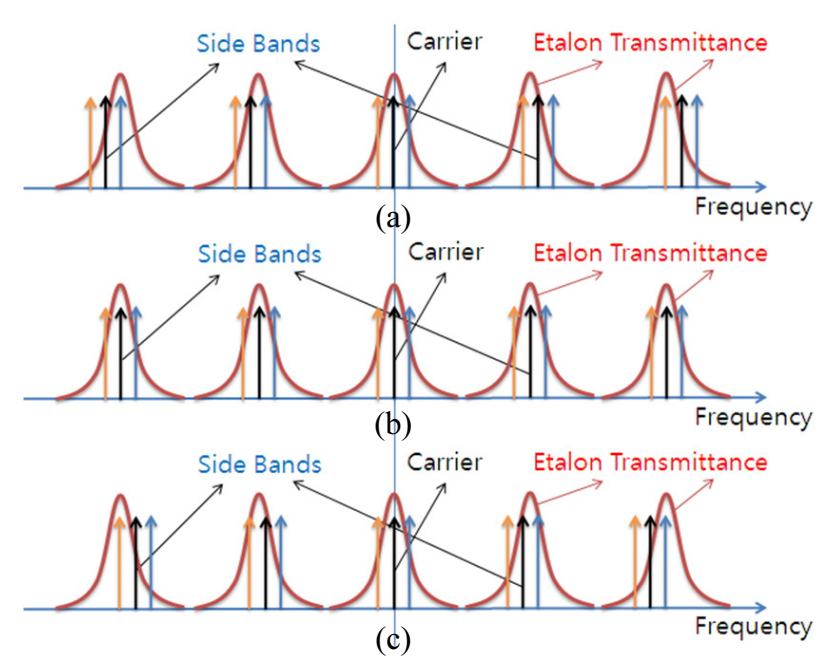

FIG. 2. Conceptual diagram of error-signal generation for the FPE-FSR measurement: (a) $\delta>0$, (b) $\delta=0$, and (c) $\delta<0$. The carrier arrow indicates the optical frequency of the probe light to be scanned at $20 \mathrm{~Hz}$, and the sideband arrows represent the sideband spectral lines generated by the sinusoidal phase modulation of the probe light. The pink curves represent the periodic transmittance of the FPE. 
curve near the probe-light (carrier) frequency, as shown in Fig. 3 (for simplicity, we only show the carrier and order $\mathrm{n}= \pm 1$ sidebands). In Fig. 3, we count the frequencydependent loss experienced by passing (or transmitting) the FPE. The sampling frequency is $\mathrm{f}_{\mathrm{FSR}}$, as implicitly shown in Fig. 3(a). As shown in Fig. 3, the frequency spacing between the mapped spectral lines becomes $\delta$. Now the mapped arrows are interpreted as the instantaneous frequencies of the sampled PM waveform. In other words, the instantaneous frequency of the sampled PM waveform swings periodically over the frequency range marked by the arrows (i.e. the frequency range between the highest-order lower and upper sidebands) in the mapped domain. Then the FPE acts as a frequency-to-amplitude converter for the sampled PM waveform, such that the instantaneous FPE output is determined by its instantaneous frequency and the FPE-TX curve (i.e. FPE finesse). For a given FPE (finesse), the error signal (i.e. mixer output in Fig. 1) depends on the amount of frequency deviation at the mapped domain: the number of sidebands, and the spacing between the mapped sidebands (i.e. $\delta$ ). Noting that the number of sidebands of the wide-band PM waveform is given by $2(\beta+1)$, we obtain the amount of frequency deviation as $2(\beta+1) \delta$ for the sampled PM waveform (i.e. in the mapped frequency domain). This means that the error signal is proportional to $2(\beta+1) \delta$.

At a middle frequency ( $v_{M}$, black arrows in Fig. 2(a)), the center (carrier) frequency is enhanced, while both the lower and upper sidebands are equally suppressed. Mapped PM spectra (after passing the FPE) are represented in Fig. 3(a) by black arrows. Envelope modulation is also observed; however, the modulation frequency is doubled (i.e. modulated

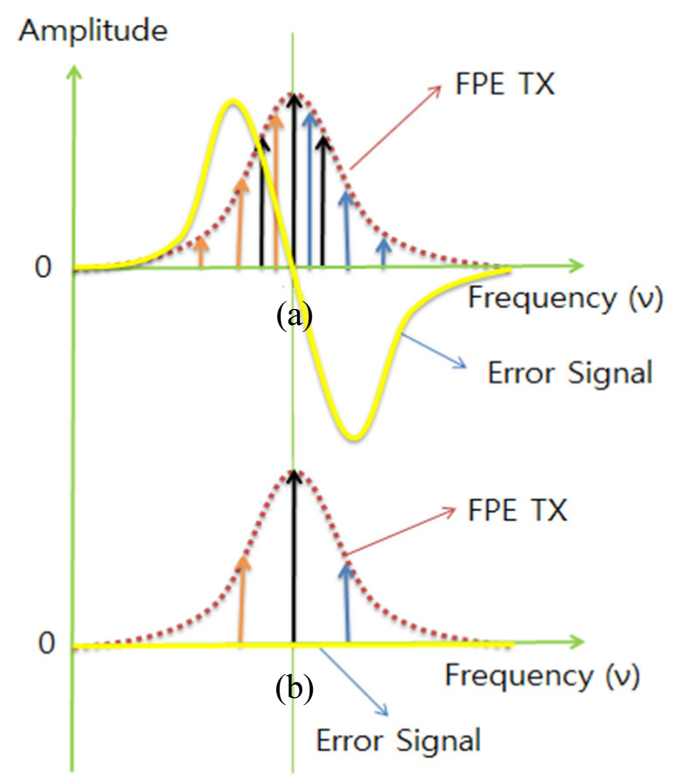

FIG. 3. Spectral mapping of the FPE-passed, PM spectral lines (arrows) and expected error signals (yellow lines): (a) $\delta$ $>0$ and (b) $\delta=0$. The amplitude of the arrows shows the frequency-dependent loss passing through the FPE. at $2 * f_{\mathrm{pm}}$ ) by swinging periodically between the lower and upper sidebands depicted in Fig. 3(a) by black arrows. Therefore, no mixer output appears. Finally, at a relatively high frequency $\left(v_{\mathrm{H}}\right.$, blue arrows in Fig. 2(a)), the upper sidebands are suppressed, while the lower sidebands are enhanced. Again, this induces envelope modulation at $f_{p m}$ of the injected PM light, but the phase is reversed compared to the case of low frequency $v_{\mathrm{L}}$ (pink arrows). At the mapped domain this is evident, as shown in Fig. 3(a).

Now we can combine all cases and predict the mixer output (i.e. error signal) as a function of $v$ (i.e. as the probe light is scanned) at a fixed $\delta$, as schematically shown by the yellow line in Fig. 3. The shape of the error signal follows approximately the first-order derivative of the FPE transmission curve. Note again that the peak-to-peak amplitude of the error signal is proportional to the frequency deviation $2(\beta+1) \delta$, as schematically shown in Fig. 3(a).

\section{2. $\delta=0\left(f_{\mathrm{pm}}=\mathbf{f}_{\mathrm{FSR}}\right)$}

Since $f_{p m}=f_{F S R}$, the carrier and sidebands of the injected PM light experience the same loss, even with scanning of the input optical frequency $v$, as shown in Fig. 2(b). At the mapped spectral domain, all the PM spectral lines overlap (no frequency deviation exists) and no envelope modulation or error signal is observed, as shown in Fig. 3(b).

\section{3. $\delta<0\left(\mathbf{f}_{\mathrm{pm}}<\mathbf{f}_{\mathrm{FSR}}\right)$}

Again, the different alignments (with respect to the FPE-TX peaks) of the PM sidebands introduce frequency-dependent loss (i.e. spectral amplitude modulation). However, the modulation phase is reversed compared to the case of $\delta>$ 0 . For example, at the probe light frequency $v_{\mathrm{H}}$ (yellow arrows in Fig. 2(c)) the upper sidebands are suppressed, while the lower sidebands are enhanced, and so on. Now we can draw the error signal at a fixed $\delta$, which is the phase-reversed shape of the case of $\delta<0$ in Fig. 3(a).

In summary, to find the exact value of the FSR $(\delta=0)$, we should sweep $f_{p m}$ toward the direction that reduces the amplitude of the error signal, and then pick the value of $\mathrm{f}_{\mathrm{pm}}$ where the amplitude becomes zero. However, in practice, it is difficult to directly find the frequency at zero amplitude, due to background and other noises. Therefore, we tune $f_{p m}$ from $f_{p m}<f_{F S R}$ to $f_{p m}>f_{F S R}$ to pass $f_{p m}=$ $f_{\mathrm{FSR}}$, then plot the amplitude of the error signal versus $f_{\mathrm{pm}}$ to find the zero-crossing point.

Noting that the mixer output $V_{\text {mix }}$ is proportional to $\delta$ and $\beta$, as discussed above, we can set up a generalized equation for the wide-band PM waveform as follows:

$$
V_{\text {mix }}(v)=V_{o} \times\{T(v+(\beta+1) \delta)-T(v-(\beta+1) \delta)\}
$$

where $V_{o}$ is a constant, and the transmittance of the FPE $T(v)$ is given by 


$$
T(v)=\frac{\left(1-R^{2}\right)^{2}}{\left(1-R^{2}\right)^{2}+4 \mathrm{R} \sin ^{2}\left(\frac{\pi v}{f_{F S R}}\right)}
$$

where $\mathrm{R}$ is the mirror reflectance of the FPE.

If $\delta$ is small, $V_{\text {mix }}(v)$ becomes

$$
V_{\text {mix }}(v)=V_{o} \times 2(\beta+1) \delta \times T^{\prime}(v)
$$

where $T^{\prime}$ is the first derivative of $T$. This clearly confirms that the error signal follows the first derivative of the FPE-TX curve, and that its peak-to-peak amplitude is proportional to $\beta, \delta$, and the maximum value of $T^{\prime}$ (i.e. FPE finesse). To improve the FSR measurement accuracy of a given FPE $\left(T^{\prime}\right)$, we aim for the strongest PM of the probe light. The wide-band PM scheme suggested here improves the measurement sensitivity by approximately a factor of $\beta$. For narrow-band PM $(\beta<1)$, equation (1) reduces to the equation given in [10].

Now let us consider the reflected light, where the FPE reflectance $R(v)=1-T(v)$. Since $R^{\prime}(v)=-T^{\prime}(v)$, the error signal becomes exactly same but with phase reversed from that of the transmitted light. As expected, no error signal appears for both transmitted and reflected signals at $\mathrm{f}_{\mathrm{pm}}=$ $\mathrm{f}_{\mathrm{FSR}}$. However, if we apply both the reflected and transmitted light that give complementary information, then we may reduce the noise and/or measurement error.

\section{EXPERIMENTAL RESULTS}

Figure 4 shows the transmittance and reflectance characteristics of the FPE used in our experiment, as a function of optical frequency $v$. Due to interference from multiple reflections, the reflectance curve shows some undulation. As discussed, the mixer output (error signal) in Fig. 1 follows approximately the first derivative of the transmission or reflection curve of the FPE (see Fig. 5). Figure 5 shows the error signals

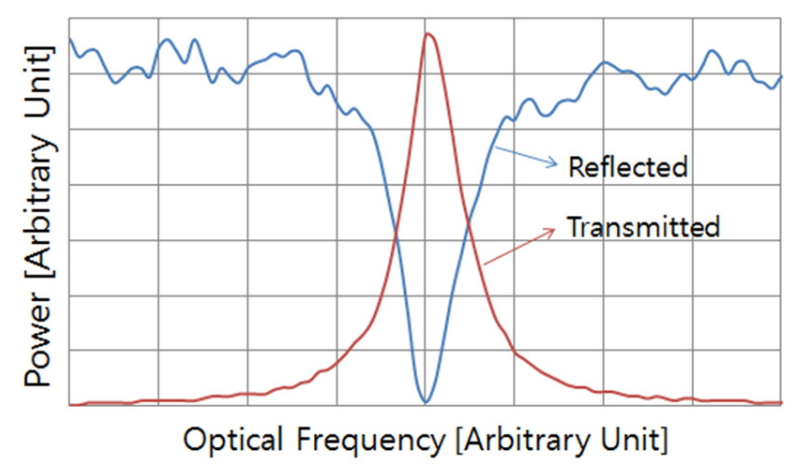

FIG. 4. Transmission (red line) and reflection (blue line) characteristics of the FPE used in our experiments. for three typical values of $\mathrm{f}_{\mathrm{pm}}$ : $\mathrm{f}_{\mathrm{pm}}=\mathrm{f}_{\mathrm{FSR}}+1 \mathrm{MHz}, \sim \mathrm{f}_{\mathrm{FSR}}$, and $\mathrm{f}_{\mathrm{FSR}}-1 \mathrm{MHz}$. For comparison, we intentionally chose the $\delta= \pm 1 \mathrm{MHz}$, corresponding to the previously reported accuracy of $100 \mathrm{ppm}$ [10] with a similar setup to ours. The error signal was averaged by the sampling scope to remove random noise. The blue and red lines represent the error signals for reflected and transmitted light respectively. As expected, the reflected and transmitted error signals (at fixed $\delta$ ) show similar shapes, but reversed phases. At $\mathrm{f}_{\mathrm{pm}}$ $\sim \mathrm{f}_{\mathrm{FSR}}$, the amplitude of the individual error signal is suppressed and becomes negligible. Passing the point $\mathrm{f}_{\mathrm{pm}}=$ $\mathrm{f}_{\mathrm{FSR}}$, both error signals have their phases reversed. We understand that the ambiguity of the error signals near $\mathrm{f}_{\mathrm{pm}}$ $=\mathrm{f}_{\mathrm{FSR}}$ is induced by the static modulation noise [10] and spectral asymmetry of the sidebands of the injected PM light.

Next, we measure the peak-to-peak amplitudes of the error signals for both transmitted and reflected light as we change $f_{p m}$, and plot the results in Fig. 6. To avoid the ambiguity of the error signals near $f_{p m}=f_{\mathrm{FSR}}$, we set the zero-crossing point of the plot as the condition for $\mathrm{f}_{\mathrm{pm}}=$ $\mathrm{f}_{\mathrm{FSR}}$. Using linear fits to the data (black straight lines), we find that the zero-crossing points for the reflected and transmitted light are $9.995765 \mathrm{GHz}$ and $9.995779 \mathrm{GHz}$ respectively. The difference may come from added noise and measurement error. We take the crossing point of both fitting lines, and find it is $9.995771 \mathrm{GHz}$. The crossing point is inherently less sensitive to background noise and/or the ambiguity of the measurement. Therefore we take the

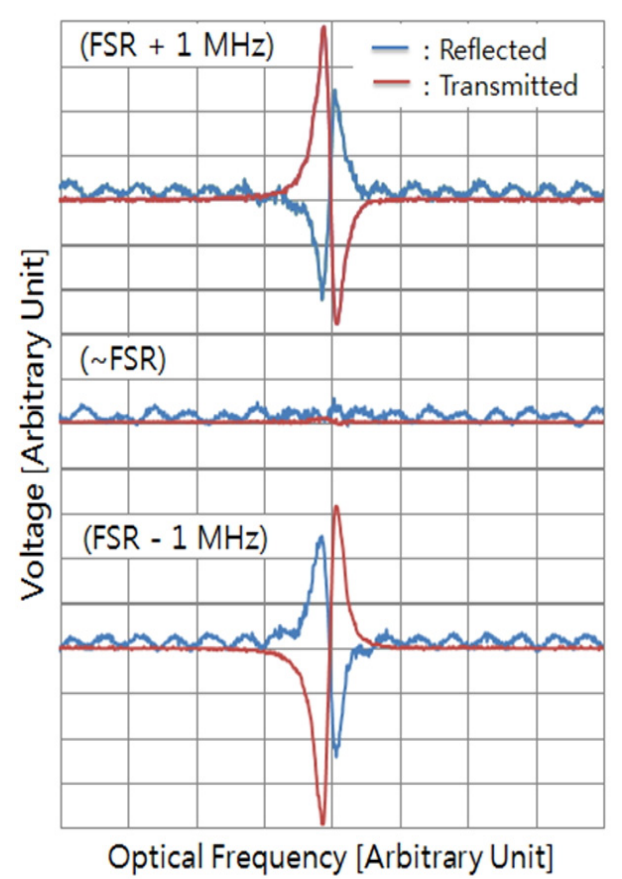

FIG. 5. Measured error signals for several values of $\mathrm{f}_{\mathrm{pm}}$ : (a) $\mathrm{f}_{\mathrm{FSR}}+1 \mathrm{MHz},(\mathrm{b}) \sim \mathrm{f}_{\mathrm{FSR}}$, and (c) $\mathrm{f}_{\mathrm{FSR}}-1 \mathrm{MHz}$. The 1-MHz deviation corresponds to the 100 -ppm accuracy previously reported result in [10]. 


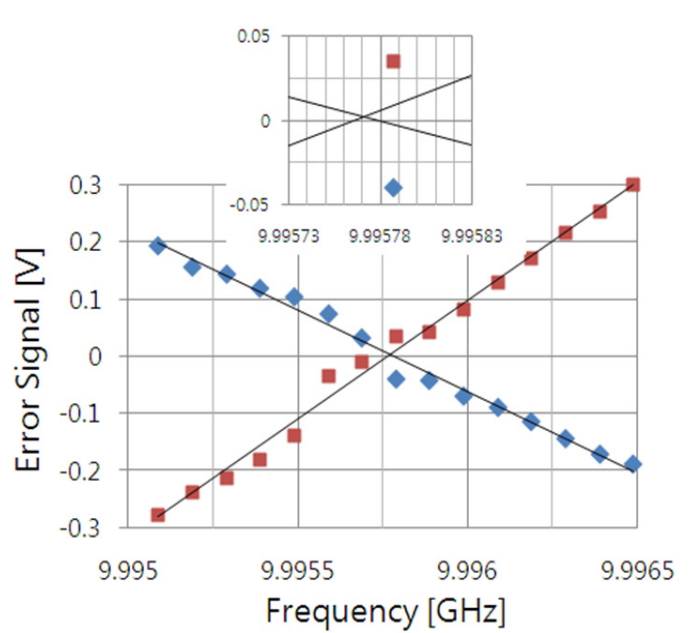

FIG. 6. Amplitude of the error signal as a function of $\mathrm{f}_{\mathrm{pm}}$. The inset (top) shows details near the zero-crossing points.

crossing point of both fitting lines as the value of $\mathrm{f}_{\mathrm{FSR}}$. Here we are able to replicate measurement within $5 \mathrm{kHz}$ accuracy, corresponding to $0.5 \mathrm{ppm}$. The two orders of magnitude in accuracy improvement over the previous result [10] is attributed mainly to the wide-band PM of the probe light, and partly to the utilization of both lights and good FPE finesse. However, phase modulation that is too strong may induce severe asymmetry in the sidebands, resulting in undesirable amplitude modulation of the PM waveform. In the peak-error-amplitude versus $f_{p m}$ plot, the data points should be on a straight line, as expected from equation (3), regardless of the polarity of $\delta$. However, due to noise and measurement error, the data groups for $\delta>0$ and for $\delta<0$ may not be on the same line, as observed in Fig. 3 in [10] and Fig. 5 in [11]. The scheme based on both reflected and transmitted light can effectively reduce the ambiguity when the data groups are not on a straight line. Further improvement of the measurement is expected by using balanced detection of the reflected and transmitted light. This may also reduce the static modulation noise mentioned in [10].

\section{CONCLUSION}

To measure the precise value of the FSR of an FPE, we used a modified Pound-Drever-Hall (PDH) method. We introduced a simple and explicit conceptual diagram of the measurement principle. Noting the periodic transmission/ reflection characteristics of an FPE, we adopted a well-known sampling theorem and developed a generalized equation for the PHD output. This equation showed that the measurement accuracy depends on the PM index and the FPE finesse. Therefore, we applied wide-band PM with $\beta=10$ (maximum value obtained in our setup) as the probe light. Previously, either the FPE reflected or (complementary) transmitted light has been used, but our measurements used both reflected and transmitted light to improve the measurement accuracy further, and we measured the FSR within $0.5 \mathrm{ppm}$. This result is two orders of magnitude better than the previous result [10] using a similar scheme. The improvement is attributed mainly to the strong PM of the probe light, and partly to the utilization of both the reflected and transmitted light and the good finesse of the FPE we employed. The suggested method using both streams of light will be more powerful when the noise significantly affects the PDH output signal, such that the PDH error data do not lie on a line.

\section{ACKNOWLEDGMENT}

This research was supported by the Basic Science Research Program through the National Research Foundation of Korea (NRF) funded by the Ministry of Education, Science and Technology (\#2011 - 0021743), and by the ICT program of MSIP/IITP, Republic of Korea (\#B0101-15-1347).

\section{REFERENCES}

1. C. H. Shin, "Optical phase-locking of semiconductor lasers," Ph. D. Dissertation (Dept. of Information Processing, Tokyo Institute of Technology, 1991).

2. I. Ozdur, D. Mandridis, N. Hoghooghi, and P. J. Delfyett, "Low noise optically tunable opto-electronic oscillator with Fabry-Perot etalon,” IEEE J. Lightwave Technol. 28, 31003106 (2010).

3. B. Batsuren, H. H. Kim, C. Y. Eom, J. J. Choi, and J. S. Lee, "Optical VSB filtering of $12.5-\mathrm{GHz}$ spaced $64 \times 12.4$ $\mathrm{Gb} / \mathrm{s}$ WDM channels using a pair of Fabry-Perot filters," J. Opt. Soc. Korea 17, 63-67 (2013).

4. J. Zhang, S. J. Hong, and D. S. Seo, "High-speed pulse train generation by spectral filtering of a mode-locked laser output," Ukr. J. of Phys. Opt. 11, 61-67 (2010).

5. K. K. Gupa and N. Onodera, "Pulse repetition frequency multiplication via intracavity optical filtering in AM modelocked fiber ring lasers," IEEE Photon. Technol. Lett. 14, 284-286 (2002).

6. K. Yiannopoulos, K. Vyrsokinos, E. Kehayas, N. Pleros, K. Vlachos, and H. Avramopoulos, "Rate multiplication by double-passing Fabry-Perot filtering," IEEE Photon. Technol. Lett. 15, 1294-1296 (2003).

7. I. Ozdur, M. Akbulut, N. Hoghooghi, D. Mandridis, S. Ozharar, F. Quinlan, and P. J. Delfyett, "A semiconductor based $10-\mathrm{GHz}$ optical comb source with sub 3-fs shot noise limited timing jitter and $\sim 500 \mathrm{~Hz}$ comb linewidth," IEEE Photon. Technol. Lett. 22, 431-433 (2010).

8. D. S. Seo, D. E. Leaird, and A. M. Weiner, "Tuning the power spectrum of a semiconductor and intracavity-etalon based mode-locked laser via detuning," Electron. Lett. 49, 1173-1175 (2013).

9. H. Jager, M. Musso, C. Neureiter, and L. Windholz, "Optical measurement of the free spectral range and spacing of plane and confocal Fabry-Perot interferometers," Opt. 
Eng. 29, 42-46 (1990).

10. S. Gee, S. Ozharar, F. Quinlan, and P. J. Delfyett, "Highprecision measurement of free spectral range of etalon," Electron. Lett. 42, 715-716 (2006).

11. I. Ozdur, S. Ozharar, F. Quinlan, S. Gee, and P. J. Delfyett, "Modified Pound-Drever-Hall scheme for high-precision free spectral range measurement of Fabry-Perot etalon," Electron.
Lett. 44, 927-928 (2008).

12. D. S. Seo, D. E. Leaird, and A. M. Weiner, "High precision measurement of free spectral range of a Fabry-Perot etalon," in Proc. The $12^{\text {th }}$ International Conference on Optical Internet (COIN2014) (Jeju, Korea, Aug. 27-29, 2014), CD, Paper FC4-3. 